\title{
Obesity and obesity-related diseases: A consequence of our man-made chemical environment?
}

\author{
Eveline Dirinck $^{1^{*}}$, Philippe Jorens ${ }^{2}$, Luc Van Gaal ${ }^{1}$ \\ ${ }^{1}$ Department of Endocrinology, Diabetology and Metabolism, Antwerp University Hospital, University of Antwerp, Edegem, Bel- \\ gium; *Corresponding Author: Eveline.Dirinck@uza.be \\ ${ }^{2}$ Department of Clinical Pharmacology/Toxicology, Antwerp University Hospital, University of Antwerp, Edegem, Belgium
}

Received 12 November 2012; revised 14 December 2012; accepted 22 December 2012

\begin{abstract}
The prevalence of obesity and its related disorders is currently attaining pandemic proportions, both in the Western and the developing world. Although lifestyle choices are commonly accepted as the main reasons, it has recently been suggested that environmental pollutants may provide an alternative cause. Several man-made chemicals have been shown to facilitate the differentiation into adipocyte at environmentally relevant levels. This process is mediated through different nuclear receptors. The endocrine function of the adipocyte itself is also affected by chemicals. This article provides a compact overview of the implicated chemicals and their modes of action. We also present the current level of evidence, linking exposure to these endocrine disrupting chemicals and obesity and its related diseases.
\end{abstract}

Keywords: Obesity; Diabetes; Endocrine Disrupting Chemicals

\section{INTRODUCTION}

The prevalence of obesity is increasing at an alarming rate throughout the world. In the United States of America, over $36 \%$ of the adult population is now estimated to be overweight [1]. The conclusion that even among US children and adolescents, obesity rates are as high as $17 \%$ is even more worrying [2]. The epidemic surpasses national borders, with similar estimates being reported in European countries and now even in the developing world $[3,4]$. Obesity is associated with a vast number of diseases, such as coronary heart disease, type 2 diabetes mellitus, some forms of cancer and even dementia [5-8]. As such, the financial cost of obesity is towering, with type 2 diabetes mellitus alone estimated to take up $10 \%$ of the health care costs in the developed world [9].

\section{ENDOCRINE DISRUPTORS}

Traditionally, the increase in obesity rates has been attributed to an increased calorie intake and a concomitant reduction in physical activity and energy expenditure [10]. However, evidence has emerged recently that other mechanisms might be involved. In 2002, Baillie-Hamilton was the first to identify the coinciding increase in the amount of man-made chemicals in our environment and the rise in obesity prevalence [11]. Indeed, after World War II, the production of synthetic organic chemicals has increased exponentially each decade. These chemicals are used in a vast array of applications, such as pesticides, components of plastics, dyes, solvents, personal care products etc. Humans are exposed to them through the ingestion of contaminated foods and drinks, the inhalation of polluted air or, in some cases, through trans-dermal absorption. As a result, the average person now has many hundreds of industrial chemicals lodged in his or her body [11]. Shortly after the large-scale introduction of these chemicals, the first reports of reproductive health issues in exposed wildlife surfaced [12]. Notably the inferior quality of eggshells and the occurrence of abnormal sex characteristics, so-called "imposex", led to dramatic decreases in certain wild life species [13,14]. These findings sparked research into the possibility of chemicals inducing endocrine disturbances. The concept "endocrine disruption" was defined as the inappropriate modulation of the endocrine system by dietary and environmental chemicals: these can interfere with the synthesis, secretion, transport, binding or action of natural hormones in the body that are responsible for the maintenance of homeostasis, reproduction, development and behavior [15]. To date, several types of chemicals have been identified as endocrine disrupting chemicals (EDC) [16]. Quite a number of these substances are very resistant to physical, chemical and biochemical degradation, and are 
therefore labeled as persistent organic pollutants (POPs) [17]. Despite the ban on production of several POPs, they are still omnipresent in our environment. Due to their persistency, these products travel the world, taken from their sites of production and use by long range atmospheric transport to remote regions of our planet, thus affecting virtually every person on earth [18]. For example the Inuit, native inhabitants of Greenland, display a serum burden of polychlorinated biphenyls (PCBs) that is a tenfold higher than their counterparts in central Europe [19].

\section{MODES OF ACTION}

\subsection{Estrogen Receptor}

Although low-dose exposure to certain chemicals has been noted to cause weight gain several decades ago, this was initially not regarded as problematic, or even a wanted side-effect, as it was in the case of anabolic steroids [11]. As such, this finding was often underreported or simply ignored. Initial concern about endocrine disruption focused on chemicals with estrogen-like activity. Although adipocytes are not considered classical targets of estrogen, they do express functional estrogen recaptors [20]. One of the first identified estrogenic endocrine disruptors is bisphenol A (BPA), a component of plastics [21]. In the presence of insulin, even very low doses of bisphenol $A$ ( $2 \mu \mathrm{g} / \mathrm{ml}$ ) are capable of stimulating the differentiation of pre-adipocytes into mature adipocytes [22]. This finding is intriguing, as it suggests that the exposure to environmental estrogens could alter the pathway of adipocyte development. Further research went on to show that prenatal and neonatal exposure to BPA increases body weight in animals $[23,24]$. Similarly, neonatal exposure of mice to tributyltin (TBT) has been shown to increase body weight and fat mass, an effect thought to be exerted, at least partially, through the estrogen receptor [25]. TBT is an organotin compound, a class of products used as antifouling agents, fungicides and heat stabilizers in plastics. Although these endocrine disruptors have been shown to exert their obesogenic affect through the estrogen recaptor, there are other, possibly more important, receptors targeted by endocrine disruptors.

\subsection{Peroxisome Proliferator-Activated Receptor Gamma}

A key factor in the process of adipogenesis is the nuclear receptor peroxisome proliferator-activatad receptor gamma (PPAR- $\gamma$ ). Its actions are a necessity in adipocyte differentiation and the identified target genes of PPAR are involved in numerous aspects of lipid metabolism and energy homeostasis [26,27]. In short, PPAR- $\gamma$ activation increases the expression of genes that promote fatty acid storage and represses genes that induce lipolysis in adipocytes in white adipose tissue [28]. PPAR- $\gamma$ belongs to the nuclear receptor family of ligand-activated transcription factors. The ligand binding pocket of PPAR- $\gamma$ is special since it is large and can accommodate a diversity of chemical structures [29]. The endogenous ligands of PPAR- $\gamma$ are diverse, including prostaglandin and linoleic acid [26]. Given the structure of its binding pocket, PPAR$\gamma$ is known to interact with several exogenous substances [29]. One of these substances is the antidiabetic drug thiazolidinedione, a drug that is linked to weight gain in humans and increased adipogenesis in cell culture [30-32]. Tributyltin is another man-made chemical capable of activating PPAR- $\gamma$. In vitro, even nanomolar levels act as activators of PPAR- $\gamma[33,34]$. A TBT exposure study demonstrated a significant dose-dependent increase in body weight of young male mice, whereas prenatal exposure to tributyltin enhances the capacity of stem cells in adult mice white adipose tissue to form adipocytes [35,36]. The last group of well-known PPAR $\gamma$ substrates are the phthalates, a group of chemicals most commonly used as softeners for plastics [37]. Mono-(2-ethylhexyl) phtalate in particular is an agonist for PPAR- $\gamma$ and selectively activates different PPAR- $\gamma$ target genes [38]. In utero exposure of mice did not result in metabolic disorders in adulthood [39]. In humans however, the 1999-2002 National Health And Nutrition Examination Survey, indicated that concentrations of several prevalent phthalate metabolites showed a statistically significant correlation with abdominal obesity and insulin resistance. The authors provided clinically interpretable data by stating that an increase in phthalate metabolites from the 10th to the 90th percentile, equaled an increase in waist circumference from 3.9 to $7.8 \mathrm{~cm}$, depending on the metabolite [40]. In the same NHANES dataset, some phthalate metabolites were associated with body mass index (BMI). These results were most consistent in men, but less so in women [41].

\subsection{Adipocytokines}

Adipose tissue itself plays an active role in energy homeostasis and is no longer perceived as a mere lipid storage compartment, but has evolved into an endocrine organ. Adipocytokines, secretagogues from the adipocytes, interact functionally with many peripheral metabolic processes. Leptin plays a major role in the control of body fat stores through coordinated regulation of feeding behaviour, metabolism, the autonomic nervous system and body energy balance [42]. Adiponectin, another recently described adipocyte-specific adipocytokine and a marker of mature adipocytes, is involved in insulin sensitivity, lipid behaviour and as such considered as the first adipocytokine being potentially protective against the diabetogenic and atherogenic aspects of adipose tissue [43]. Several known EDC have been identified to influence adi- 
pocytokine levels. Prenatal exposure of rats to diisobutyl phthalate reduced leptin levels in both sexes while perinatal exposure of rats to low doses of Bisphenol A predisposes to hyperleptinemia in adulthood [44,45]. This is accompanied by a severe metabolic syndrome, including obesity, dyslipidemia, hyperglycemia, hyperinsulinemia, and glucose intolerance [45]. Bisphenol A is also capable of inhibiting adiponectin release from human adipocytes in vitro [46]. Taken together, exposure to EDC might cause a metabolically unfavorable secretagogue profile of the adipocyte.

\section{EARLY LIFE EXPOSURE}

Metabolic programming of critical tissues such as the adipose tissue mainly takes place during foetal development. As reviewed by Heindel and vom Saal, external effects on the developing foetus might permanently alter metabolic pathways that are crucial for energy homeostasis and thus alter the "setpoint" for developing obesity later in life [47]. Nicotine use provides a fine example of this theory, termed the developmental origins of adult disease (DOAD) [48]. Smoking during pregnancy causes direct effects, such as intrauterine growth restriction and low birth weight, but it also predisposes to weight gain later in life. [49]

\section{HUMAN HEALTH AFFECTED BY ENDOCRINE DISRUPTORS}

\subsection{Obesity}

In vitro data and animal studies provide us with mechanistic data on the obesogenic effects of endocrine disruptors. Population-based epidemiological studies evaluat0 ing associations between various endocrine disruptors and obesity have been reviewed recently [50]. Particularly in the field of persistent organic pollutants, there have been numerous studies published on the possible influence of exposure to EDC early in life and the development of obesity. Positive associations have been reported, although some of these results have been difficult to replicate. For example in utero exposure to pesticides such as DDT has been linked to future obesity, but not in every cohort [51, 52]. Cross-sectional studies of adult exposure to certain POPs have yielded data suggestive of a positive relation. In particular the NHANES database has offered a unique opportunity, linking exposure to PCBs and several organochlorine pesticides to obesity. [53] On the other hand, we among many other groups have published a significant negative correlation between BMI, waist, fat mass percentage, total and subcutaneous abdominal adipose tissue, and serum levels of some EDCs such as PCBs [54]. Combined, these results suggest that the effect of lowdose exposure to POPs might be more complicated than a simple obesogenic effect.

\subsection{Type 2 Diabetes Mellitus, the Metabolic Syndrome and Cardiovascular Disease}

Several of the EDCs considered to be obesogenic, have been implicated in the development of type 2 diabetes mellitus as well, one of the most important obesity-related diseases. The mechanisms by which these EDC cause type 2 diabetes mellitus surpass their mere obesogenic effect. In vitro and animal data have been accumulated suggesting a direct effect on the pancreatic beta-cell [55]. One of the first and most notorious reports suggesting a possible link between exposure to chemicals and type 2 diabetes mellitus has been published by the US National Academy of Sciences' Institute of Medicine on the US Air Force veterans of operation Ranch Hand, responsible for spraying defoliants during the Vietnam War. This cohort displays a slight but consistent increase in diabetes incidence among subjects with elevated pesticide serum concentrations, as well as glucose and/or insulin abnormalities among all exposed individuals [56]. Ensuing studies, in particular the ones using the database of NHANES, have also implicated other OCPs and PCBs in the development of type 2 diabetes [57-59]. This suggests that even non-occupational, low exposure can trigger a disruption of the glucose metabolism. Even more compelling is the finding that serum levels of different EDCs are capable of predicting the development of diabetes after 20 years, as well as other dysmetabolic conditions [60]. Cross-sectional analysis of the NHANES database also indicates a clear link between several EDCs (organochlorine pesticides and polychlorinated biphenyls) and the occurrence of the metabolic syndrome in nondiabetic individuals [61]. The potential implication of EDCs in the development of cardiovascular risk factors requires further research, considering the health burden caused by cardiovascular diseases.

\section{SPECIFIC DIFFICULTIES RELATED TO THE STUDY OF EDC}

Interpreting the available human data on the link between endocrine disrupting chemicals and obesity and its related diseases is challenging for numerous reasons.

- The ultimate outcome, whether it is BMI, weight or diabetes, is affected by a near infinite list of life style choices (nutritional composition of the diet, exercising habits, smoking, medication...). Scrupulous data collection is necessary in order to be able to correct for these influences.

- Some of the detected positive associations are confined to subgroups, mainly determined by gender [62]. Therefore large cohorts, enabling good subgroup analysis, need to be recruited. 
- Most studies focus on several EDCs, thus filtering out the effect of an individual compound can be difficult. On the other hand, since the levels of POPs tend to be strongly correlated, it is plausible that it is actually the synergistic effect of several substances that causes the ultimate observed effect.

- The interpretation of cross-sectional study results with POPs offers particular difficulties, since the metabolism of POPs is different between obese and lean individuals, with adiposity delaying the metabolism and increasing the half-life of these chemicals. In general, there are very limited data on the kinetics of EDC after absorption.

- Unlike the more traditional toxicology, EDCs do not always follow a monotone dose-response relationship. Some EDCs seem to cause a strong effect at low, environmentally relevant doses, but a weakened or no effect at high doses. This inverted U-shape association has been suggested by some experimental studies and further observed in epidemiological studies [59, 63]. This is problematic, as a linear dose-response relationship is traditionally regarded as a criterion for causality by epidemiologists.

- As suggested by the developmental origins of adult disease (DOAD) theory, the time elapsing between exposure to the EDC and the development of obesity can extend over many years. Careful assessment of pregnant women and a very prolonged follow-up of their offspring will be necessary to unravel the late consequences of early-life exposure.

- Finally, EDCs can induce epigenetic changes, which are transmitted to progenity [64]. Therefore, an individual's susceptibility to obesogenic influences in his/ her environment, may actually be an inherited trait, caused by EDC exposure of an ancestor.

\section{CONCLUSION}

The emerging role of endocrine disrupting chemicals in the current pandemic of obesity and obesity-related diseases is an exciting new area of interest. The presented findings are beginning to shift the focus from the simplistic notion that obesity can be understood and controlled only by focusing of energy intake and expenditure. A vast number of questions regarding modes of action remain to be elucidated, and this warrants urgent further research. The collection of data on human exposure and their effects is as crucial, despite the fact that field is burdened with a puzzling complexity. Present data indicate a greater vulnerability to the detrimental effects of EDC in the neonatal phase. The weight of the current evidence is enough to prompt a precautionary attitude towards EDC. Given the ubiquitous dissimination of EDCs, protecting ourselves and our offspring will require international col- laboration on a multitude of levels.

\section{REFERENCES}

[1] Flegal, K.M., et al. (2012) Prevalence of obesity and trends in the distribution of body mass index among US adults, 1999-2010. JAMA, 307, 491-497. doi:10.1001/jama.2012.39

[2] Ogden, C.L., et al. (2012) Prevalence of obesity and trends in body mass index among US children and adolescents, 1999-2010. JAMA, 307, 483-90.

[3] Seidell, J.C. (2000) Obesity, insulin resistance and diabetes-A worldwide epidemic. British Journal of Nutrition, 83, S5-S8.

[4] Han, J.C., et al. (2010) Childhood obesity. Lancet, 375, 1737-1748.

[5] Van Gaal, L.F., et al. (2006) Mechanisms linking obesity with cardiovascular disease. Nature, 444, 875-880

[6] Cameron, A.J. et al. (2012) A systematic review of the impact of including both waist and hip circumference in risk models for cardiovascular diseases, diabetes and mortality. Obesity Reviews, 41, 484-494.

[7] Yehuda-Shnaidman, E., et al. (2012) Mechanisms linking obesity, inflammation and altered metabolism to colon carcinogenesis. Obesity Reviews, 13, 1083-1095.

[8] Lee, E.B. (2011) Obesity, leptin, and Alzheimer's disease. Annals of the New York Academy of Sciences, 1243, 1529.

[9] Rayman, G. and Kilvert, A. (2012) The crisis in diabetes care in England. BMJ, 15, e5446.

[10] Prentice, A.M. (2001) Overeating: The health risks. Obesity Research, 9, 234S-238S. doi:10.1038/oby.2001.124

[11] Baillie-Hamilton, P.F. (2002) Chemical toxins: A hypothesis to explain the global obesity epidemic. The Journal of Alternative and Complementary Medicine, 8, 185-192.

[12] Smith, B.S. (1981) Reproductive anomalies in stenoglossan snails related to pollution from marinas. Journal of Applied Toxicology, 1, 15-21.

[13] Smith, B.S. (1981) Male characteristics on female mud snails caused by antifouling bottom paints. Journal of Applied Toxicology, 1, 22-25.

[14] Matthiessen, P., et al. (1995) Changes in periwinkle (Littorina littorea) populations following the ban on TBT-based antifoulings on small boats in the United Kingdom. Ecotoxicology and Environmental Safety, 30, 180-194.

[15] Vos, J.G., et al. (2000) Health effects of endocrine-disrupting chemicals on wildlife, with special reference to the European situation. Critical Reviews in Toxicology, 30, 71-133. doi:10.1080/10408440091159176

[16] Sung, E., et al. (2012) Detection of endocrine disruptorsfrom simple assays to whole genome scanning. International Journal of Andrology, 35, 407-414.

[17] Ballschmite, K., et al. (2002) Man-made chemicals found in remote areas of the world: The experimental definition for POPs. Environmental Science and Pollution Research, 9, 274-88. doi:10.1007/BF02987503 
[18] Breivik, K., et al. (2006) Empirical and modeling evidence of the long-range atmospheric transport of decabromodiphenyl ether. Environmental Science \& Technology, 40, 4612-4618.

[19] Toft, G., et al. (2008) Menstrual cycle characteristics in European and Inuit women exposed to persistent organochlorine pollutants. Human Reproduction, 23, 193-200.

[20] Pedersen, S.B., et al. (2004) Estrogen controls lipolysis by up-regulating alpha2A-adrenergic receptors directly in human adipose tissue through the estrogen receptor alpha. Implications for the female fat distribution. The Journal of Clinical Endocrinology \& Metabolism, 89, 1869-1878.

[21] Krishnan, A.V., et al. (1993) Bisphenol-A: An estrogenic substance is released from polycarbonate flasks during autoclaving. Endocrinology, 132, 2279-2286.

[22] Masuno, H., et al. (2001) Bisphenol A in combination with insulin can accelerate the conversion of 3T3-L1 fibroblasts to adipocytes. The Journal of Lipid Research, 43, 676-684.

[23] Rubin, B.S., et al. (2001) Perinatal exposure to low doses of bisphenol A affects body weight, patterns of estrous cyclicity, and plasma LH levels. Environmental Health Perspectives, 109, 675-680.

[24] Somm, E., et al. (2009) Perinatal exposure to bisphenol a alters early adipogenesis in the rat. Environmental Health Perspectives, 117, 1549-1555.

[25] Penza, M., et al. (2011) The environmental chemical tributyltin chloride (TBT) shows both estrogenic and adipogenic activities in mice which might depend on the exposure dose. Toxicology and Applied Pharmacology, 255, 65-75.

[26] Rosen, E.D., et al. (1999) PPAR gamma is required for the differentiation of adipose tissue in vivo and in vitro. Molecular Cell, 4, 611-617.

[27] Tontonoz, P. and Spiegelman, B.M. (2008) Fat and beyond: The diverse biology of PPARgamma. Annual Review of Biochemistry, 77, 289-312. doi:10.1146/annurev.biochem.77.061307.091829

[28] Ferré, P. (2004) The biology of peroxisome proliferatoractivated receptors: Relationship with lipid metabolism and insulin sensitivity. Diabetes, 53, S43-S50.

[29] Maloney, E.K. and Waxman, D.J. (1999) Trans-activation of PPARalpha and PPARgamma by structurally diverse environmental chemicals. Toxicology and Applied Pharmacology, 161, 209-218.

[30] Lehmann, J.M. (1995) An antidiabetic thiazolidinedione is a high affinity ligand for peroxisome proliferator-activated receptor gamma (PPAR gamma). The Journal of Biological Chemistry, 270, 12953-12956.

[31] Liu, S.C. (2012) Effect of antidiabetic agents added to metformin on glycaemic control, hypoglycaemia and weight change in patients with type 2 diabetes: A network meta-analysis. Diabetes, Obesity and Metabolism, 14, 810820.

[32] Tchoukalova, Y.D., et al. (2012) In vivo adipogenesis in rats measured by cell kinetics in adipocytes and plasticadherent stroma-vascular cells in response to high-fat diet and thiazolidinedione. Diabetes, 61, 137-144.
[33] Kanayama, T., et al. (2005) Organotin compounds promote adipocyte differentiation as agonists of the peroxisome proliferator-activated receptor gamma/retinoid X receptor pathway. Molecular Pharmacology, 67, 766-774.

[34] Inadera, H. and Shimomura, A. (2005) Environmental chemical tributyltin augments adipocyte differentiation. Toxicology Letters, 159, 226-234.

[35] Zuo, Z., et al. (2011) Tributyltin causes obesity and hepatic steatosis in male mice. Environmental Toxicology, 26, 79-85.

[36] Kirchner, S., et al. (2010) Prenatal exposure to the environmental obesogen tributyltin predisposes multipotent stem cells to become adipocytes. Molecular Endocrinology, 24, 526-539.

[37] Bility, M.T., et al. (2004) Activation of mouse and human peroxisome proliferator-activated receptors (PPARs) by phthalate monoesters. Society of Toxicology, 82, 170-182. doi:10.1093/toxsci/kfh253

[38] Feige, J.N., et al. (2007) The endocrine disruptor monoethyl-hexyl-phthalate is a selective peroxisome proliferator-activated receptor gamma modulator that promotes adipogenesis. The Journal of Biological Chemistry, 282, 19152-19166. doi:10.1074/jbc.M702724200

[39] Casals-Casas, C., et al. (2008) Interference of pollutants with PPARs: Endocrine disruption meets metabolism. International Journal of Obesity, 32, S53-S61. doi:10.1038/ijo.2008.207

[40] Stahlhut, R.W., et al. (2007) Concentrations of urinary phthalate metabolites are associated with increased waist circumference and insulin resistance in adult US males. Environmental Health Perspectives, 115, 876-882.

[41] Hatch, E.E., et al. (2008) Association of urinary phthalate metabolite concentrations with body mass index and waist circumference: A cross-sectional study of NHANES data, 1999-2002. Environmental Health, 3, 27.

[42] Wauters, M., et al. (2001) Polymorphisms in the leptin receptor gene, body composition and fat distribution in overweight and obese women. International Journal of Obesity and Related Metabolic Disorders, 25, 714-720.

[43] Turer, A.T. and Scherer, P.E. (2012) Adiponectin: Mechanistic insights and clinical implications. Diabetologia, 55, 2319-2326.

[44] Boberg, J., et al. (2008) Impact of diisobutyl phthalate and other PPAR agonists on steroidogenesis and plasma insulin and leptin levels in fetal rats. Toxicology, 250, 7581.

[45] Wei, J., et al. (2011) Perinatal exposure to bisphenol A at reference dose predisposes offspring to metabolic syndrome in adult rats on a high-fat diet. Endocrinology, 152, 3049-3061.

[46] Hugo, E.R., et al. (2008) Bisphenol A at environmentally relevant doses inhibits adiponectin release from human adipose tissue explants and adipocytes. Environmental Health Perspectives, 116, 1642-1647.

[47] Heindel, J.J. and vom Saal, F.S. (2009) Role of nutrition and environmental endocrine disrupting chemicals during the perinatal period on the aetiology of obesity. Molecular and Cellular Endocrinology, 304, 90-96. 
[48] Newbold, R.R., et al. (2007) Developmental exposure to endocrine disruptors and the obesity epidemic. Reproductive Toxicology, 23, 290-296. doi:10.1016/j.reprotox.2006.12.010

[49] Beyerlein, A., et al (2011) Is low birth weight in the causal pathway of the association between maternal smoking in pregnancy and higher BMI in the offspring? European Journal of Epidemiology, 26, 413-420.

[50] Tang-Péronard, J.L., et al. (2011) Endocrine-disrupting chemicals and obesity development in humans: A review. Obesity Reviews, 12, 622-636.

[51] Karmaus, W., et al. (2009) Maternal levels of dichlorodiphenyl-dichloroethylene (DDE) may increase weight and body mass index in adult female offspring. Occupational and Environmental Medicine, 66, 143-149.

[52] Gladen, B.C., et al. (2004) Prenatal DDT exposure in relation to anthropometric and pubertal measures in adolescent males. Environmental Health Perspectives, 112, 17611767.

[53] Elobeid, M.A., et al. (2010) Endocrine disruptors and obesity: An examination of selected persistent organic pollutants in the NHANES 1999-2002 data. International Journal of Environmental Research and Public Health, 7, 2988-3005.

[54] Dirinck, E., et al. (2011) Obesity and persistent organic pollutants: Possible obesogenic effect of organochlorine pesticides and polychlorinated biphenyls. Obesity, 19, 709-714.

[55] Hectors, T.L., et al. (2011) Environmental pollutants and type 2 diabetes: A review of mechanisms that can disrupt beta cell function. Diabetologia, 54, 1273-1290.

[56] Henriksen, G.L., et al. (1997) Serum dioxin and diabetes mellitus in veterans of Operation Ranch Hand. Epidemio$\log y, \mathbf{8}, 252-258$.

[57] Everett, C.J., et al. (2007) Association of a polychlori- nated dibenzo-p-dioxin, a polychlorinated biphenyl, and DDT with diabetes in the 1999-2002 National Health and Nutrition Examination Survey. Environmental Research, 103, 413-418.

[58] Lee, D.-H., et al. (2006) A strong dose-response relation between serum concentrations of persistent organic pollutants and diabetes: Results from the National Health and Examination Survey 1999-2002. Diabetes Care, 29, 16381644. doi:10.2337/dc06-0543

[59] Lee, D.-H., et al. (2007) Extended analysis of the association between serum concentrations of persistent organic pollutants and diabetes. Diabetes Care, 30, 15961598. doi:10.2337/dc07-0072

[60] Lee, D.-H., et al. (2010) Low dose of some persistent organic pollutants predicts type 2 diabetes: A nested casecontrol study. Environmental Health Perspectives, 118, 1235-1242. doi:10.1289/ehp.0901480

[61] Lee, D.H., et al. (2007) Relationship between serum concentrations of persistent organic pollutants and the prevalence of metabolic syndrome among non-diabetic adults: results from the National Health and Nutrition Examination Survey 1999-2002. Diabetologia, 50, 1841-1851.

[62] Gladen, B.C., et al. (2000) Pubertal growth and development and prenatal and lactational exposure to polychlorinated biphenyls and dichlorodiphenyl dichloroethene. Journal of Pediatrics, 136, 490-496. doi:10.1016/S0022-3476(00)90012-X

[63] Welshons, W.V., et al. (2006) Large effects from small exposures. III. Endocrine mechanisms mediating effects of bisphenol A at levels of human exposure. Endocrinol$o g y$, 147, S56-S69. doi:10.1210/en.2005-1159

[64] Wolstenholme, J.T., et al. (2012) Gestational exposure to bisphenol a produces transgenerational changes in behaviors and gene expression. Endocrinology, 153, 3828-3838. 